\title{
Application of the GLUE Method to parameter uncertainty analysis of Xin'anjiang model
}

\author{
Chao Zhang ${ }^{1,}$, , Rubin Wang ${ }^{2, b}$ \\ ${ }^{1}$ Research Institute of Geotechnical Engineering, Hohai University, Nanjing 210098, China \\ ${ }^{2}$ Key Laboratory of Ministry of Education for Geomechanics and Embankment Engineering, Hohai \\ University, Nanjing 210098, China

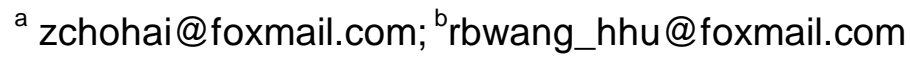

Keywords: Hydrological Model; GLUE method; Xin'anjiang model; Uncertainty analysis; Rainfall-runoff Simulation

Abstract. Uncertainty analysis of the parameters of conceptual rainfall-runoff models is studied via application of the Xin'anjiang (XAJ) model as a case study. A new research methodology is suggested. We suggest to fix a subset of insensitive parameters first, and then analyze the uncertainty of the other parameters. The results show that after fixing some insensitive parameters, the scatter plot of parameters $K, S M$, and $C S$ exhibit likelihood values with higher slopes and display more obvious clustering phenomenon in the daily rainfall-runoff simulation. It is inferred that the influence of insensitive parameters is one of the factors contributing to hydrological forecasting uncertainty.

\section{Introduction}

The parameters of conceptual rainfall-runoff models reflect watershed hydrological characteristics. In theory, the value of parameters of the Xin' anjiang (XAJ) model [1] could be directly measured since all of the parameters have definite physical meaning. However, in practice, a lack of the measurement and experiment often requires that they be calibrated as a set of "optimal" parameters by using a system identification method based on observation data[2-3]. The uncertainty of model parameter calibration will lead to uncertainty in the hydrological process simulation and forecast result. At present, the quantitative description of parameter calibration uncertainty and its influence on hydrologic forecasting uncertainty has become a popular topic of research.

There is always uncertainty in watershed rainfall-runoff models. A novel approach to parameter uncertainty analysis of hydrological models using neural networks is developed by Shrestha et al.[4]. The key aspect we are focused on when applying these models is how the uncertainty will affect the precision and effective use of the model. The Generalized Likelihood Uncertainty Estimation (GLUE) [5] is one analysis method that attempts to quantify uncertainty from this perspective. Because of its simplicity, ease of use, and ability to simulate complex problems, the GLUE method has been successfully applied in the field of hydrology.

The XAJ model is used extensively throughout the world; however, there is little research to date on its parameter uncertainty. On the basis of previous studies, we attempt to conduct further research.

\section{Requirements of GLUE}

The results calculated by the GLUE method largely depend on the choice of likelihood function. The results presented in this study make use of the sum of squared errors[6] as the likelihood function, in the form:

$$
L\left(\underline{\theta}_{i} \mid \underline{Y}\right)=\left(1-\sigma_{i}^{2} / \sigma_{o b s}^{2}\right)^{N} ; \sigma_{i}^{2}<\sigma_{o b s}^{2}
$$

where $L\left(\underline{\theta}_{i} \mid \underline{Y}\right)$ is the likelihood function value for the $i$ th model conditioned on the observational data, $\sigma_{i}^{2}$ is the associated error variance for the $i$ th model, $\sigma_{o b s}^{2}$ is the observed variance for the period 
under consideration, and $N$ is a parameter. Results will be presented herein for different values of $N$, and also for the function:

$$
L\left(\underline{\theta}_{i} \mid \underline{Y}\right)=\exp \left\{-N \sigma_{i}^{2} / \sigma_{o b s}^{2}\right\} ; \sigma_{i}^{2}<\sigma_{o b s}^{2}
$$

which has the feature that in applying Bayes' equation for updating of the likelihood weights, the error variance for each period of data is given equal weight[6].

\section{Definition of the Parameters Ranges of XAJ Model}

The GLUE methodology is used to calibrate the XAJ model using a continuous 7-year record (1981-1987) of historical data for the Yandu River watershed $\left(601 \mathrm{~km}^{2}\right)$, China. The parameters of the model are shown in Table 1, together with the respective ranges. We conduct 50,000 sets of parameters sampling via uniform distribution. These ranges were chosen on the basis of physical meaning. These ranges are wider than the expected possible values for the watershed to ensure that the simulation could cover the factual data and that a random combination of parameters could fully represent the watershed. However, all prior likelihood evaluations are set to 0 for parameter values outside these parameter ranges.

Table 1 Parameter search ranges of XAJ Model

\begin{tabular}{cccccccccccccc}
\hline Parameters & $K$ & $B$ & $C$ & $W M$ & $W U M$ & $W L M$ & $S M$ & $E X$ & $K I$ & $C S$ & $C I$ & $C G$ & $I M P$ \\
\hline Lower bound & 0.1 & 0.1 & 0.1 & 80 & 10 & 30 & 1 & 0.1 & 0.01 & 0.01 & 0.5 & 0.5 & 0.001 \\
Upper bound & 2 & 0.9 & 0.9 & 200 & 100 & 100 & 100 & 2 & 0.7 & 0.9 & 0.999 & 0.999 & 0.05 \\
\hline
\end{tabular}

where, $K$ :Ratio of potential evapotranspiration topan evaporation; WM: Areal mean tension water capacity; WUM: Upper layer areal mean tension water capacity; WLM: Lower layer areal mean tension water capacity; $C$ : Coefficient of deep evapotranspiration; IMP: Ratio of impervious area to total area of basin; $B$ : Exponent of the tension water capacity curve; $S M$ : Areal mean of the free water capacity of the surface soil layer; $E X$ : Exponent of free water capacity curve; $K I$ : Outflow coefficients of free water storage to interflow relationships; $K G$ : Outflow coefficients of free water storage to groundwater relationships; $C S$ : Recession constant for routing through the channel system within each subbasin; $C I$ : Recession constant of the lower interflow storage; $C G$ : Recession constant of groundwater; $N$ : Number of subreaches; $K E$ : Travel time of flood wave through subreach; $X E$ : Flow ration of subreaches.

\section{Application of GLUE to the XAJ Model - - Flood simulation}

We selected 9 floods that occurred during the period of 1981 to 1987 from historical data on the Yandu River for this study. The top 6 floods are used to calculate the parameters, and the other 3 floods are used for verification and validation. There were 30000 sets of parameter sampling that were taken by uniform distribution.

Sensitivity analysis of XAJ Model parameters. Sensitivity analysis of parameters in the flood simulation is similar to the process taken in the daily rainfall-runoff simulation. Fig. 1 shows the scatter plot for parameters of Flood 19830623. As can be seen from Fig.1, the parameter $K$, which is sensitive in the daily rainfall-runoff simulation, becomes insensitive in the flood simulation. The parameter $S M$ is varies markedly between $(15,25)$. It can be inferred that is sensitive within this interval and has a remarkable uncertainty. The parameters $W M, W U M$, and $W L M$ tend to decrease within their search interval, and this indicates that they are relatively sensitive within the Yandu River watershed. The parameter $C G$ has a small influence on flood simulation. The other parameters have little influence in the flood simulation.

Updating of Uncertainty Bounds. Updating of the likelihood distribution as additional data becomes available may be achieved by the application of Bayes' equation in the following form:

$$
L\left(\underline{\mathrm{Y}} \mid \underline{\theta}_{i}\right)=L\left(\underline{\theta}_{i} \mid \underline{Y}\right) L_{o}\left(\underline{\theta}_{i}\right) / C
$$


where $L_{o}\left(\underline{\theta}_{i}\right)$ is a prior likelihood for the parameter set $\underline{\theta}, L\left(\underline{\theta}_{i} \mid \underline{Y}\right)$ is the likelihood function calculated with the set of observed variables $\underline{Y}, L\left(\underline{\mathrm{Y}} \mid \underline{\theta}_{i}\right)$ is the posterior likelihood for the simulation of $\underline{Y}$ given $\underline{\theta}$, and $C$ is a scaling constant calculated such that the cumulative value of $L\left(\underline{\mathrm{Y}} \underline{\theta}_{i}\right)$ equals one. Given the results from the first period of simulation, the resulting posterior distribution can be used as the prior distribution for the next period. If further observational data from the next period are available, the resulting likelihood values can be used with Equation (3) to update the posterior likelihood distribution for any further predictions [6].
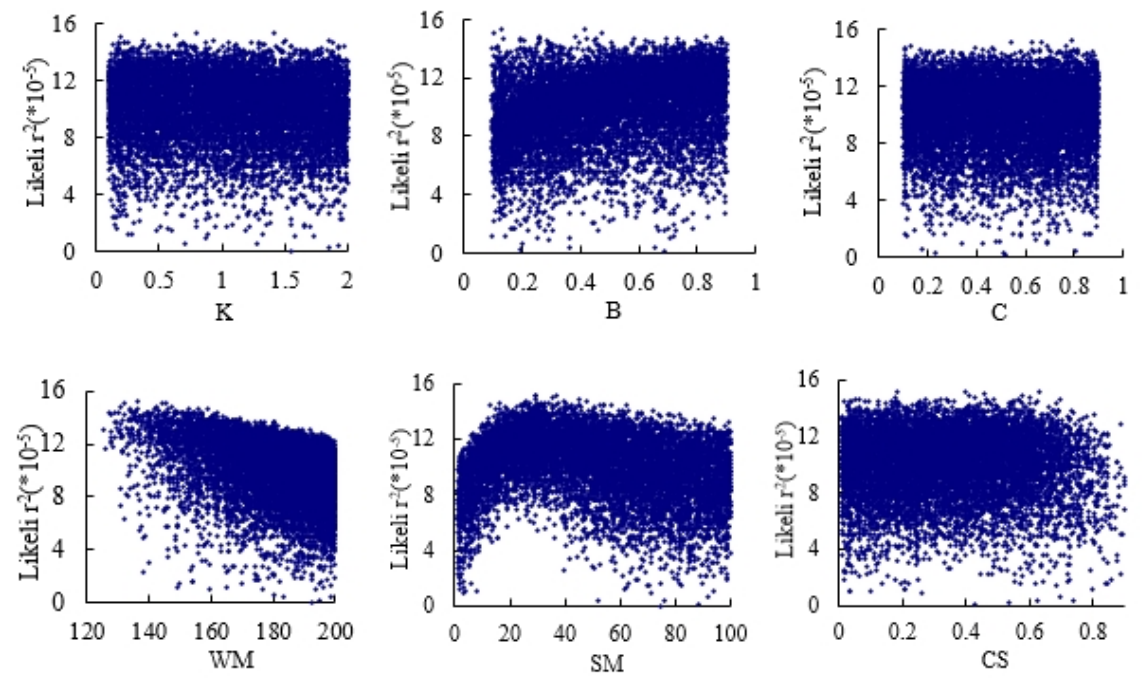

Fig.1. Scatter plot for parameters in the flood simulation of Flood 19830623

Equation (3) is used to update the distribution of likelihood values with observation data. Fig.2 shows the uncertainty bounds for 6 floods. Figures on the left show the results of prior distributions being used, while figures on the right show the results of posterior distributions being used.

As can be seen from Figure 10, the observed discharge is generally contained within the calculated uncertainty bounds. This illustrates that the application of the XAJ model to Yandu River is feasible. The uncertainty bounds calculated by posterior distributions updated using the observation data tended to be narrower and contained more observed discharges compared to those calculated by prior distributions. This suggests that the forecast precision is improved after considering uncertainty. 

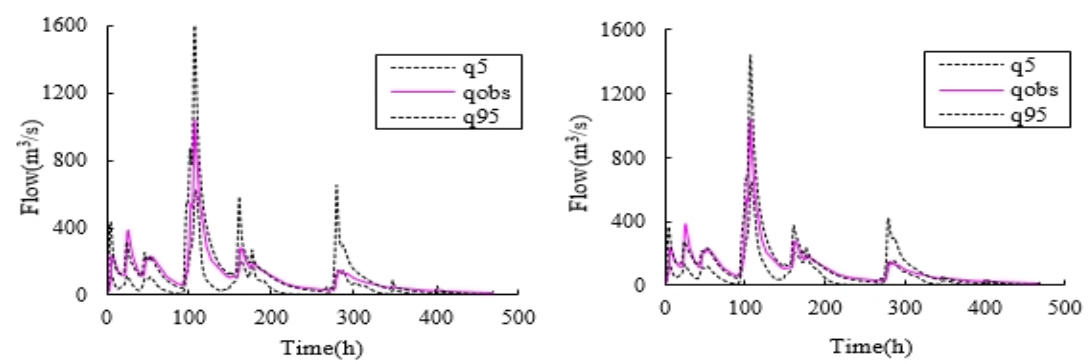

(a) Flood 19820716
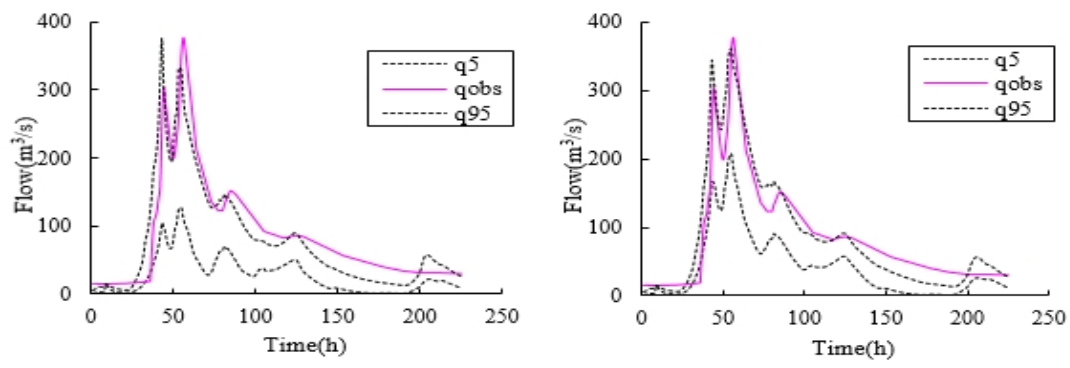

(b) Flood 19831017
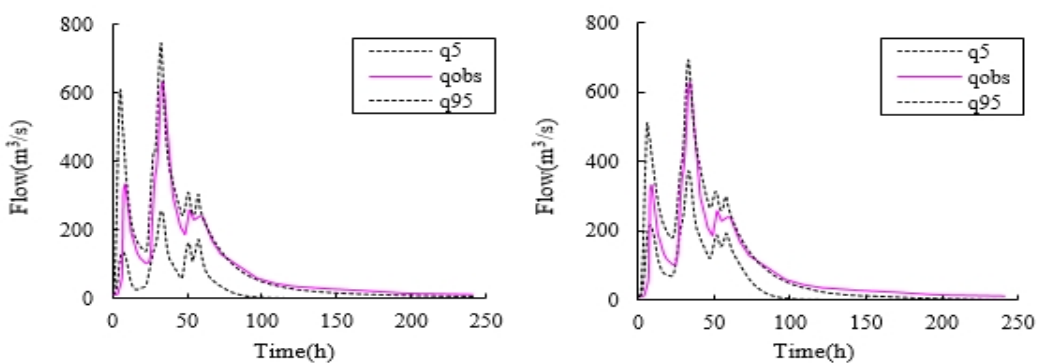

(c) Flood 19840612
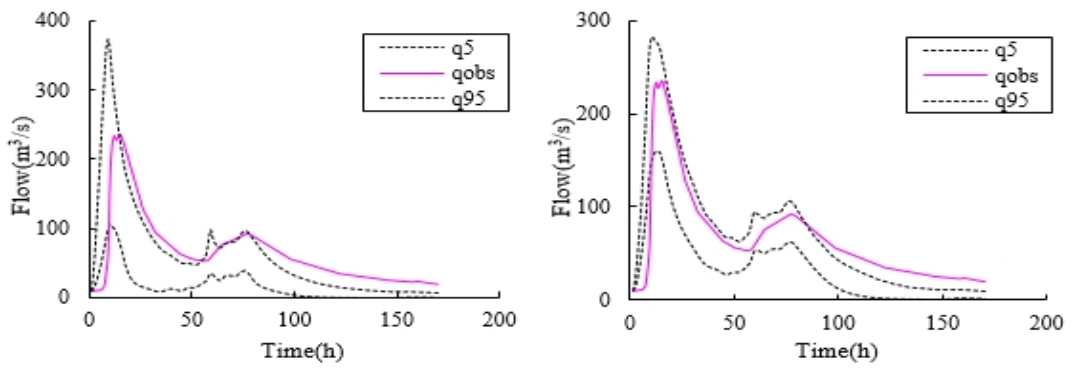

(d) Flood 19840703
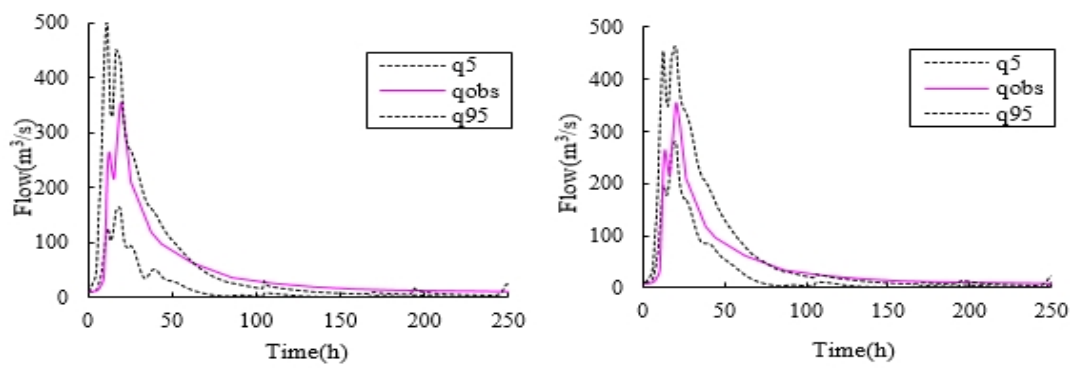

(e) Flood 19840909
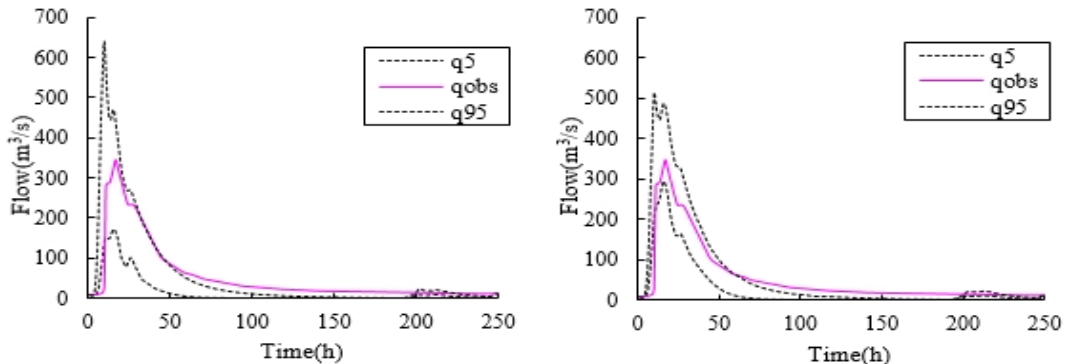

(e) Flood 19850424

Fig. 2. $90 \%$ uncertainty bounds for 6 floods. 
Model validation. When using GLUE methodology, the posterior distributions calculated can be directly used to evaluate the uncertainty bounds of a future event without observed data. The method could also be used to validate the model through observed data which have not been used in updates. Fig.3. shows the uncertainty bounds of 3 floods obtained from posterior distributions for 12 floods in the Yandu River watershed.

As can be seen from Fig.3, the effect of model validation for most of the floods is good. Most of the observed discharges fall inside the $90 \%$ uncertainty bounds. It can be inferred that likelihood distributions calculated from the top 6 floods can be used for flood forecasting and can obtain a reasonable forecasting interval.

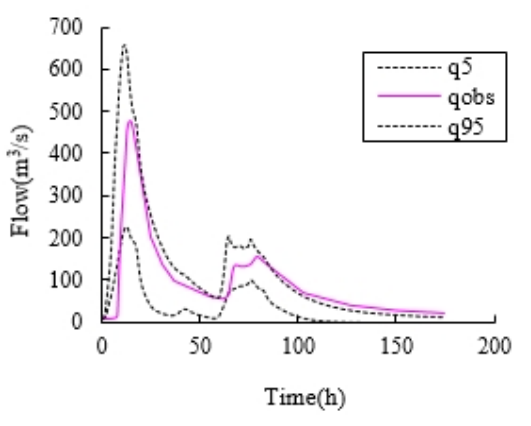

(a) Flood 19850621

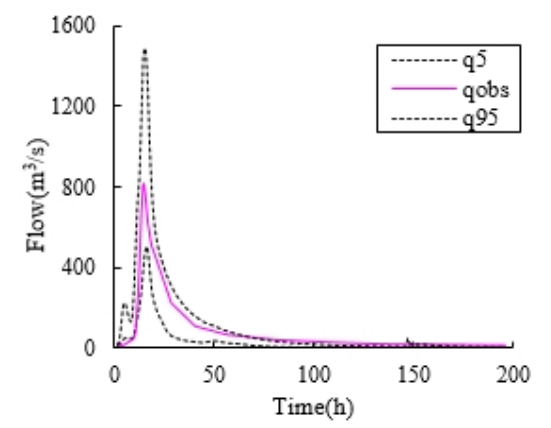

(b) Flood 19870719

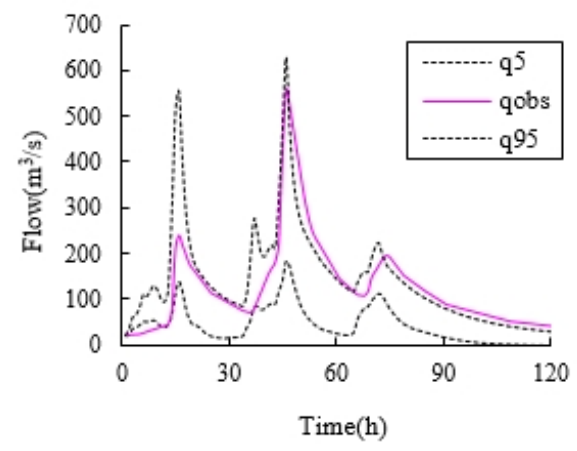

(c) Flood 19870821

Fig.3. $90 \%$ uncertainty bounds for 3 floods.

\section{Conclusions}

A new research methodology is presented which involves fixing some insensitive parameters and then analyzing the uncertainty of the other parameters. The results show that after eliminating the influence of insensitive parameters, the parameter "equifinality" phenomenon is still evident. It is inferred that a comprehensive evaluation of the uncertainty of hydrological forecasting cannot be made only through parameter sensitivity analysis. The results show that the scatter plot of parameters K, SM, and CS exhibit likelihood values with higher slopes and exhibit more obvious clustering in the daily rainfall-runoff simulation. Also, the parameter uncertainty has a tendency to reduce. The $90 \%$ uncertainty bounds of model forecast are obviously narrowed and could more properly contain the observed runoff process. It is inferred that the influence of insensitive parameters is one of the factors impacting hydrological forecasting uncertainty.

\section{Acknowledgements}

This work was financially supported by the National Natural Science Foundation of China (No.51409082), and China Postdoctoral Science Foundation (2013M531264). 


\section{References}

[1] R.J. Zhao: Journal of Hydrology Vol.135(1992), p.371.

[2] C. Zhang, R.B. Wang, and Q.X. Meng: Advances in Meteorology, Article ID 545376 (2015), p.1.

[3] V.A. Cooper, V.T.A. Nguyen and J.A. Nicell: Journal of Hydrology, Vol.334 (2007), p.455.

[4] D.L. Shrestha, N. Kayastha, and D.P. Solomatine: Hydrol. Earth Syst. Sci. Vol.13(2009), p.1235.

[5] K. Beven and A. Binley: Hydrological Processes, Vol. 6 (1992), p. 279.

[6] J. Freer and K. Beven: Water Resources Research, Vol. 32 (1996), p.2161. 